\title{
Impaired chloroplast positioning affects photosynthetic capacity and regulation of the central carbohydrate metabolism during cold acclimation
}

\author{
Anastasia Kitashova ${ }^{1}\left[\right.$ [ Katja Schneider ${ }^{2} \cdot$ Lisa Fürtauer $^{1} \mathbb{D} \cdot$ Laura Schröder $^{1} \cdot$ Tim Scheibenbogen $^{1}(\mathbb{D}$. \\ Siegfried Fürtauer ${ }^{1,3}$ (D) Thomas Nägele ${ }^{1}$ (i)
}

Received: 8 May 2020 / Accepted: 6 November 2020 / Published online: 19 November 2020

(c) The Author(s) 2020

\begin{abstract}
Photosynthesis and carbohydrate metabolism of higher plants need to be tightly regulated to prevent tissue damage during environmental changes. The intracellular position of chloroplasts changes due to a changing light regime. Chloroplast avoidance and accumulation response under high and low light, respectively, are well known phenomena, and deficiency of chloroplast movement has been shown to result in photodamage and reduced biomass accumulation. Yet, effects of chloroplast positioning on underlying metabolic regulation are less well understood. Here, we analysed photosynthesis together with metabolites and enzyme activities of the central carbohydrate metabolism during cold acclimation of the chloroplast unusual positioning 1 (chup1) mutant of Arabidopsis thaliana. We compared cold acclimation under ambient and low light and found that maximum quantum yield of PSII was significantly lower in chup 1 than in Col-0 under both conditions. Our findings indicated that net $\mathrm{CO}_{2}$ assimilation in chupl is rather limited by biochemistry than by photochemistry. Further, coldinduced dynamics of sucrose phosphate synthase differed significantly between both genotypes. Together with a reduced rate of sucrose cycling derived from kinetic model simulations our study provides evidence for a central role of chloroplast positioning for photosynthetic and metabolic acclimation to low temperature.
\end{abstract}

Keywords Cold acclimation · Chloroplast positioning · Photosynthesis · Arabidopsis thaliana $\cdot$ Carbohydrate metabolism · Hexose phosphates $\cdot$ Hexokinase $\cdot$ Kinetic modelling

\section{Introduction}

Tight regulation and balance of photosynthetic primary and secondary reactions are a prerequisite for efficient $\mathrm{CO}_{2}$ fixation, biomass accumulation and stress tolerance of higher plants. Particularly under changing environmental

Electronic supplementary material The online version of this article (https://doi.org/10.1007/s11120-020-00795-y) contains supplementary material, which is available to authorized users.

Thomas Nägele

thomas.naegele@1mu.de

1 Department Biology I, Plant Evolutionary Cell Biology, LMU München, 82152 Planegg-Martinsried, Germany

2 Department Biology I, Plant Development, LMU München, 82152 Planegg-Martinsried, Germany

3 Fraunhofer Institute for Process Engineering and Packaging IVV, 85354 Freising, Germany conditions, stabilization of this balance is essential for prevention of tissue damage due to cytotoxic accumulation of reactive oxygen species (ROS). Intracellular distribution of chloroplasts changes in response to changing light intensity. Chloroplast movement plays an important role in protection of photosynthetic machinery against high light intensities and increases photosynthetic efficiency under low light (Wada 2013). Typically, high light induces an avoidance response where chloroplasts are located at the side-walls of palisade cells to minimize light absorption. Under low light, chloroplasts re-distribute to the upper and lower side of the cells to maximize light absorption (accumulation response; schematic overview in (Wada 2013)).

The cytoplasmic protein CHLORPLAST UNUSUAL POSITIONING 1 (CHUP1) localizes to the chloroplast surface, contains an actin binding domain and has been shown to be essential for chloroplast movement (Oikawa et al. 2003, 2008). It was demonstrated that CHUP1 interacts with actin, independent of its filamentation status, and 
further binds to profilin which is an actin modifying protein (Schmidt von Braun and Schleiff 2008). On gene expression level, no alteration of other components involved in chloroplast positioning was observed in a CHUP1 deletion mutant (Schmidt von Braun and Schleiff 2008). From their gene expression data, the authors concluded that the observed genetic effect rather resulted from altered positioning of chloroplasts and peroxisomes, which was shown to be coupled before (Oikawa et al. 2003), than from alteration of genes encoding for components of light perception and chloroplast movement. Under short-day growth conditions, i.e. $8 \mathrm{~h}$ light phase, chupl plants displayed reduced photosynthesis and biomass accumulation over a wide range of light intensities, i.e. $50-300 \mu \mathrm{mol} \mathrm{m}{ }^{-2} \mathrm{~s}^{-1}$ (Gotoh et al. 2018). In their study, Gotoh and co-workers compared different mutants affected in chloroplast positioning and finally concluded that trade-offs of biomass production and photoprotection are essentially driven by chloroplast movement under changing growth light.

With decreasing temperature, changes in light intensity become an increasing challenge for plants. During the multigenic process of cold acclimation, which is induced by exposure of higher plants to low but non-freezing temperature, numerous molecular and physiological processes are adjusted to enhance freezing tolerance and to minimize cellular and tissue damage, e.g. caused by ROS production (Dreyer and Dietz 2018; Levitt 1980; Xin and Browse 2000). Cold acclimation plays an important role in ecology and evolution of many plant species of the temperate zone due to the significant impact of low temperature on their range boundaries (Hoffmann 2002). It affects gene expression, translational, post-translational and metabolic regulation (Bahrani et al. 2019; Fürtauer et al. 2019; Liu et al. 2019; Wang et al. 2017). Further, composition and activation state of the photosynthetic apparatus is adjusted within a process termed photosynthetic acclimation, in which, however, many of the involved molecular mechanisms still remain elusive (Herrmann et al. 2019). Low temperature is generally accompanied by a reduced membrane fluidity which affects, e.g., the repair cycle of the D1 protein (Aro et al. 1990). Additionally, low temperature significantly affects enzyme activities of the Calvin-Benson cycle which needs to be counteracted during cold stress response and acclimation to prevent imbalance between primary and secondary photosynthetic reactions (Khanal et al. 2017). Absorption of excessive sunlight by the light-harvesting complex leads to ROS generation which damages the photosystems, particularly PSII, and finally leads to photoinhibition (Gururani Mayank et al. 2015). Yet, while particularly under low temperature excessive light is harmful for plants, a combination of low temperature and light is essential for enhancing freezing tolerance in Arabidopsis thaliana (Wanner and Junttila 1999).
Comparing temperature effects on photosynthesis in natural Arabidopsis accessions previously revealed that limitation of photosynthesis by triose-phosphate utilization was less under low temperature than under high temperature comparing low vs. high irradiance (Pons 2012). Together with many other studies, this observation clearly emphasizes the essential role of tight regulation of photosynthesis and carbohydrate metabolism. Sucrose (Suc) metabolism is well known to play a central role in photosynthetic acclimation (Herrmann et al. 2019; Nägele et al. 2012; Stitt et al. 2010; Strand et al. 2003). In the light, photosynthetic $\mathrm{CO}_{2}$ fixation results in triose phosphates which are substrate for starch biosynthesis in the chloroplast and Suc biosynthesis in the cytosol. Key regulators of Suc biosynthesis are cytosolic fructose-1,6-bisphosphatase (cFBPase) and sucrose phosphate synthase (SPS) (Strand et al. 2000). Suc is the central transport sugar in many plant species, represents an osmotically active solute and is involved in sugar signalling (Ruan 2014). Supportingly, recent findings suggest that Suc export from the chloroplast into the cytosol is involved in cold acclimation and is required for development of maximal freezing tolerance (Patzke et al. 2019). Also, in our own studies we demonstrated that Suc compartmentation and invertase-driven cleavage in cytosol and vacuole significantly affect cold stress response and stabilization of photosynthesis (Nägele and Heyer 2013; Weiszmann et al. 2018).

In the present study we aimed to reveal the effect of defective chloroplast positioning on photosynthesis and regulation of carbohydrate metabolism during cold acclimation under ambient and low light in Arabidopsis thaliana.

\section{Materials and methods}

\section{Plant material and growth conditions}

Plants of Arabidopsis thaliana accession Col-0 and the homozygous chupl T-DNA insertion line SALK_129128C (locus At3g25690; validated by PCR) were grown on a 1:1 mixture of GS90 soil and vermiculite in a climate chamber under short-day conditions $(8 \mathrm{~h} / 16 \mathrm{~h}$ light/dark; $100 \mu \mathrm{mol} \mathrm{m}{ }^{-2} \mathrm{~s}^{-1} ; 22{ }^{\circ} \mathrm{C} / 16{ }^{\circ} \mathrm{C} ; 60 \%$ relative humidity). After 6 weeks, plants were transferred to the greenhouse

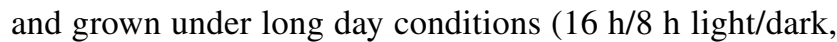
$100 \mu \mathrm{mol} \mathrm{m} \mathrm{m}^{-2} \mathrm{~s}^{-1}$ ). After 5 days in the greenhouse, plants were either (i) sampled at midday, i.e. after $8 \mathrm{~h}$ in the light, or (ii) transferred to a cold room for cold acclimation at $4{ }^{\circ} \mathrm{C}$ under long day conditions ( $16 \mathrm{~h} / 8 \mathrm{~h}$ light/dark), with either PAR $100 \mu \mathrm{mol} \mathrm{m}{ }^{-2} \mathrm{~s}^{-1}$ (samples ambient light/low temperature, AL/LT) or PAR $20 \mu \mathrm{mol} \mathrm{m}{ }^{-2} \mathrm{~s}^{-1}$ (samples low light/low temperature, LL/LT). After 7 days at $4{ }^{\circ} \mathrm{C}$, plants were sampled at midday after $8 \mathrm{~h}$ in the light. One sample consisted of 3 leaf rosettes which were immediately frozen 
in liquid nitrogen and stored at $-80^{\circ} \mathrm{C}$ until further use. For photosynthesis measurements, plants were grown with the same protocol.

\section{Net photosynthesis and chlorophyll fluorescence measurements}

Maximum quantum yield of PSII ( $\mathrm{Fv} / \mathrm{Fm})$ was determined after $15 \mathrm{~min}$ of dark adaptation by supplying a saturating light pulse. Dynamics of quantum efficiency of PSII (ФPSII), electron transport rate (ETR), photochemical (qP), non-photochemical quenching ( $\mathrm{qN}$ ) and rates of net $\mathrm{CO}_{2}$ assimilation, i.e. net photosynthesis (NPS), were determined within light response curves by stepwise increase of photosynthetically active radiation (PAR) from 0 to $1200 \mu \mathrm{mol} \mathrm{m}^{-2} \mathrm{~s}^{-1}$ in 5 min intervals. Additionally, all parameters were recorded within $\mathrm{CO}_{2}$ response curves where $\mathrm{CO}_{2}$ concentration was stepwise increased from 50 to $1200 \mathrm{ppm}$ under constant PAR intensity of $1200 \mu \mathrm{mol} \mathrm{m} \mathrm{m}^{-2} \mathrm{~s}^{-1}$. All measurements were performed using a WALZ® GFS-3000FL system equipped with measurement head 3010-S and Arabidopsis chamber 3010-A (Heinz Walz GmbH, Effeltrich, Germany, https://www.walz. com/). NPS and chlorophyll fluorescence parameters of control plants were determined at ambient temperature $\left(22^{\circ} \mathrm{C}\right)$, parameters of $\mathrm{AL} / \mathrm{LT}$ and LL/LT plants were determined at $4{ }^{\circ} \mathrm{C}$.

\section{Quantification of amount of starch, soluble carbohydrates and hexose phosphates}

Starch amount was determined as described previously with slight modification (Nägele et al. 2012). $400 \mu \mathrm{l}$ of $80 \%$ $\mathrm{EtOH}$ were added to ground plant material and incubated at $80{ }^{\circ} \mathrm{C}$ for $30 \mathrm{~min}$. After centrifugation, the supernatant was transferred into a new tube and extraction was repeated. Supernatants (80\% EtOH) were pooled, dried in a desiccator and used for quantification of soluble carbohydrates. Starch containing pellets were incubated with $500 \mu \mathrm{l} 0.5 \mathrm{~N}$ $\mathrm{NaOH}$ at $95{ }^{\circ} \mathrm{C}$ for 60 min before slight acidification with $500 \mu \mathrm{l} 1 \mathrm{M} \mathrm{CH}_{3} \mathrm{COOH}$. The starch hydrolysate was digested with amyloglucosidase for $2 \mathrm{~h}$ at $55{ }^{\circ} \mathrm{C}$. Glucose content, derived from starch digestion, was photometrically determined by a glucose oxidase/peroxidase/o-dianisidine reaction at $540 \mathrm{~nm}$.

Soluble sugars, i.e. glucose, fructose and sucrose, were determined from dried EtOH extracts. Sucrose was quantified using an anthrone assay after incubation with $30 \% \mathrm{KOH}$ at $95{ }^{\circ} \mathrm{C}$. For the assay, anthrone was dissolved in $14.6 \mathrm{M}$ $\mathrm{H}_{2} \mathrm{SO}_{4}(0.14 \% \mathrm{w} / \mathrm{v})$, incubated with the samples for $30 \mathrm{~min}$ at $40{ }^{\circ} \mathrm{C}$ before absorbance was determined at $620 \mathrm{~nm}$.

Glucose amount was determined using a coupled hexokinase/glucose 6-phosphate dehydrogenase assay resulting in $\mathrm{NADPH}+\mathrm{H}^{+}$which was determined photometrically at $340 \mathrm{~nm}$. For fructose quantification, phosphoglucoisomerase was added to the reaction mixture after glucose determination.

Glucose 6-phosphate (G6P) and fructose 6-phosphate (F6P) were extracted and photometrically quantified using enzyme cycling-based assays as described earlier (Gibon et al. 2002). In brief, hexose phosphates were extracted using trichloroacetic acid (TCA) in diethyl ether ( $16 \% \mathrm{w} / \mathrm{v})$, washed with $16 \%(\mathrm{w} / \mathrm{v})$ TCA in $5 \mathrm{mM}$ EGTA and neutralised with $5 \mathrm{M} \mathrm{KOH} / 1 \mathrm{M}$ triethanolamine. Enzymatic reactions catalysed the equimolar interconversion of G6P and F6P into $\mathrm{NADPH}+\mathrm{H}^{+}$which was finally detected photometrically from slopes of a cyclic reaction yielding a formazan dye from thiazolyl blue (MTT) at $570 \mathrm{~nm}$.

\section{Quantification of enzyme activities}

Activity of all enzymes was determined under substrate saturation $\left(v_{\max }\right)$.

Activity of sucrose phosphate synthase (SPS) was determined using the anthrone assay as described earlier (Nägele et al. 2012). Frozen leaf tissue was suspended in extraction buffer containing $50 \mathrm{mM}$ HEPES-KOH (pH 7.5), $10 \mathrm{mM}$ $\mathrm{MgCl}_{2}, 1 \mathrm{mM}$ EDTA, $2.5 \mathrm{mM}$ DTT, $10 \%$ (v/v) glycerine and $0.1 \%(\mathrm{v} / \mathrm{v})$ Triton-X-100. After incubation on ice, extracts were incubated for $30 \mathrm{~min}$ at $25{ }^{\circ} \mathrm{C}$ with reaction buffer containing $50 \mathrm{mM}$ HEPES-KOH (pH 7.5), $15 \mathrm{mM}$ $\mathrm{MgCl}_{2}, 2.5 \mathrm{mM}$ DTT, $35 \mathrm{mM}$ UDP-glucose, $35 \mathrm{mM}$ F6P and $140 \mathrm{mM}$ G6P. Reactions were stopped by adding $30 \% \mathrm{KOH}$ and heating the samples to $95{ }^{\circ} \mathrm{C}$. Sucrose was determined photometrically after incubation with anthrone in $\mathrm{H}_{2} \mathrm{SO}_{4}$ as described above and blanks (stopped before reaction) were subtracted.

Gluco- and fructokinase activities were determined photometrically at $340 \mathrm{~nm}$ recording the slopes of $\mathrm{NADP}^{+}$ reduction to $\mathrm{NADPH}+\mathrm{H}^{+}$(Wiese et al. 1999). In brief, frozen leaf tissue was suspended in extraction buffer containing $50 \mathrm{mM}$ Tris- $\mathrm{HCl}$ (pH 8.0), $0.5 \mathrm{mM} \mathrm{MgCl} 2,1 \mathrm{mM}$ EDTA, $1 \mathrm{mM}$ DTT and $1 \%(\mathrm{v} / \mathrm{v})$ Triton-X-100. Following incubation on ice, reaction was started with a reaction buffer containing $100 \mathrm{mM}$ HEPES-KOH (pH 7.5), $10 \mathrm{mM} \mathrm{MgCl}_{2}$, $2 \mathrm{mM}$ ATP, $1 \mathrm{mM}$ NADP, 0.5U G6PDH and either $5 \mathrm{mM}$ glucose for glucokinase measurement or $5 \mathrm{mM}$ fructose and 0.5U PGI for fructokinase measurement.

Neutral (nInv), acidic (aInv) and cell wall-bound (cwInv) invertase activities were determined as described before with slight modifications (Nägele et al. 2010). Frozen leaf tissue was homogenized in extraction buffer containing $50 \mathrm{mM}$ HEPES-KOH (pH 7.5), 5 mM MgCl, 2 mM EDTA, 1 mM phenylmethylsulfonylfluoride (PMSF), $1 \mathrm{mM}$ DTT, 10\% $(\mathrm{v} / \mathrm{v})$ glycerine and $0.1 \%(\mathrm{v} / \mathrm{v})$ Triton-X-100. After incubation on ice, samples were centrifuged at $4{ }^{\circ} \mathrm{C}$ and supernatants were transferred to a new tube. Pellets contained 
cell wall-bound invertase and were re-suspended in extraction buffer. Soluble, i.e. nInv and aInv, invertase activities were determined from supernatants, cwInv activity was determined from resuspended pellets. Activity of nInv was determined using a reaction buffer with $\mathrm{pH} 7.5(20 \mathrm{mM}$ HEPES-KOH, $100 \mathrm{mM}$ sucrose), aInv and cwInv activities were determined at $\mathrm{pH} 4.7$ (20 mM sodium acetate, $100 \mathrm{mM}$ sucrose). After incubation of extract with reaction buffers reactions were stopped at $95{ }^{\circ} \mathrm{C}$ and glucose built from invertase reactions was photometrically determined by a glucose oxidase/peroxidase/o-dianisidine assay at $540 \mathrm{~nm}$.

Activity of glucose 6-phosphate dehydrogenase (G6PDH) under substrate saturation was determined as reported earlier (Fahrendorf et al. 1995) with slight modification. Ground plant material was suspended in extraction buffer consisting of $0.05 \mathrm{M}$ Tris $\mathrm{HCl}(\mathrm{pH} 8.0), 0.3 \mathrm{M} \mathrm{NaCl}, 0.1 \mathrm{mM}$ benzamidine, $0.1 \mathrm{mM}$ PMSF, $1 \mathrm{mM}$ DTT and $0.1 \%(\mathrm{v} / \mathrm{v})$ Triton$\mathrm{X}-100$. A low concentration of $1 \mathrm{mM}$ DTT was used due to the previous observation of a potential reduction of G6PDH activity in mature spinach leaves and barley primary leaves by $10 \mathrm{mM}$ DTT (Johnson 1972). After incubation on ice and centrifugation at $4{ }^{\circ} \mathrm{C}$, supernatants were incubated with assay buffer containing $0.1 \mathrm{M}$ Tris $\mathrm{HCl}(\mathrm{pH} 8.0)$ and $0.4 \mathrm{mM}$ NADP. After pre-incubation at $30{ }^{\circ} \mathrm{C}$ for $10 \mathrm{~min}$, G6P was added and reduction of $\mathrm{NADP}^{+}$by G6PDH was measured photometrically at $340 \mathrm{~nm}$ at $30^{\circ} \mathrm{C}$ for $10 \mathrm{~min}$.

Phosphoglucose isomerase (PGI) extraction and activity quantification under substrate saturation was based on previously described methods with slight modification ( Kunz et al. 2014). Ground plant material was suspended in extraction buffer containing $0.05 \mathrm{M}$ Tris $\mathrm{HCl}(\mathrm{pH} 8.0)$, $0.5 \mathrm{mM} \mathrm{MgCl} 2,1 \mathrm{mM}$ EDTA, $2.5 \mathrm{mM}$ DTT and $1 \%(\mathrm{v} / \mathrm{v})$ Triton-X-100. The suspension was incubated on ice, followed by centrifugation at $4{ }^{\circ} \mathrm{C}$. PGI activity was estimated from supernatants in a coupled reaction with G6PDH by photometrically measuring reduction of $\mathrm{NADP}^{+}$at $340 \mathrm{~nm}$. For this, supernatants were incubated with assay buffer consisting of $100 \mathrm{mM}$ HEPES-KOH (pH 7.5) and $10 \mathrm{mM}$ $\mathrm{MgCl}_{2}$. After addition of $2 \mu \mathrm{l}$ of $100 \mathrm{mM} \mathrm{NADP}$ and $1 \mathrm{U}$ $\mathrm{G} 6 \mathrm{PDH}$, the solution was pre-incubated at $30{ }^{\circ} \mathrm{C}$ for $10 \mathrm{~min}$. The reaction was initiated by addition of $20 \mu \mathrm{l}$ of $50 \mathrm{mM}$ F6P (or $20 \mu \mathrm{l}$ of $\mathrm{ddH}_{2} \mathrm{O}$ for blanks), followed by recording absorbance at $340 \mathrm{~nm}$ at $30{ }^{\circ} \mathrm{C}$ for $10 \mathrm{~min}$. The linear range of recorded kinetic was interpolated and the slope was used to calculate maximal activity of PGI.

\section{Sample preparation and sectioning for microscopy analysis}

Primary leaves of Arabidopsis thaliana, Col-0 and chupl, were cut into $1 \mathrm{~mm}$ square pieces and fixed in fixation buffer (75 mM sodium cacodylate, $2 \mathrm{mM} \mathrm{MgCl}_{2}, \mathrm{pH}$ 7) substituted with $2.5 \%(\mathrm{v} / \mathrm{v})$ glutaraldehyde for 2 days at $4{ }^{\circ} \mathrm{C}$. The leave pieces were washed three times at room temperature using fixation buffer and post-fixed with $1 \%(\mathrm{w} / \mathrm{v})$ osmium tetroxide for $2 \mathrm{~h}$. Subsequent to washing with fixation buffer and water, the samples were stained en bloc with $1 \%(\mathrm{w} / \mathrm{v})$ uranyl acetate in $20 \%(\mathrm{v} / \mathrm{v})$ acetone for $30 \mathrm{~min}$. Samples were dehydrated in a series of graded acetone and embedded in Spurr's resin. Samples were either ultra-thin sectioned (thickness, $60 \mathrm{~nm}$ ) for TEM (transmission electron microscope) analysis or semi-thin sectioned (thickness, $2 \mu \mathrm{m}$ ) for LM (light microscope) analysis. For sectioning a diamond knife was used on a Reichert Ultracut-E ultramicrotome. Ultra-thin sections were mounted on collodium-coated copper grids, post-stained with lead citrate $(80 \mathrm{mM}, \mathrm{pH}$ 13) and examined with an EM 912 transmission electron microscope (Zeiss, Oberkochen, Germany) equipped with an integrated OMEGA energy filter operated in the zeroloss mode at $80 \mathrm{kV}$. Images were acquired using a $2 \mathrm{k} \times 2 \mathrm{k}$ slow-scan CCD camera (Tröndle Restlichtverstärkersysteme, Moorenweis, Germany). Semi-thin sections mounted on glass slides and examined using a Zeiss Axiophot light microscope. Images were acquired using a SPOT insight 2 MP CCD color digital camera.

\section{Statistics and mathematical modelling}

For statistical data evaluation we used the free software environment R Version 3.6.1 (https://www.r-project.org/) (R Core Team 2019) and RStudio Version 1.2.5019 (https ://rstudio.com/) (RStudio Team 2019). Statistics comprised analysis of variance (ANOVA) and Tukey's honestly significant difference post-hoc test. Mathematical modelling was performed in MATLAB ${ }^{\circledR}$ R2019b (www.mathworks. com) with the toolbox IQM Tools developed by IntiQuan (https://www.intiquan.com/). Ordinary differential equations (ODEs), enzyme kinetic equations and kinetic parameter values are provided in the supplements (Supplementary Table IV-VI). Rates of net photosynthesis ('rNPS') were derived from interpolation of photosynthesis measurements, i.e. net $\mathrm{CO}_{2}$ assimilation rates (interpolation model: $f(x)=$ $\left.a^{*} \exp \left(b^{*} x\right)+c^{*} \exp \left(d^{*} x\right)\right)$. For simulation of sucrose cleavage rates, experimentally quantified invertase activities of nInv, aInv and cwInv were summed up (model parameter 'vmax_inv'). Rates of net starch synthesis ('rStarch') were estimated from the amount measured after $8 \mathrm{~h}$ in the light (i.e. amount $/ 8$ h). For simulation at low temperature (AL/LT and LL/LT), quantified $v_{\max }$ values were temperature corrected to $4{ }^{\circ} \mathrm{C}$ applying the van't Hoff rule (Eq. 1) with a $\mathrm{Q}_{10}$ factor of 2.5 (Reyes et al. 2008).

$Q_{10}=(R 2 / R 1)^{\frac{10 K}{(T 2-T 1)}}$

Ranges of kinetic parameters which were not experimentally quantified in the present study, i.e. $K_{M}$ and $K_{i}$ values, 
were derived from our previous studies and further literature (Fürtauer and Nägele 2016; Nägele et al. 2010, 2012; Scharte et al. 2009; Schnarrenberger and Oeser 1974; Weiszmann et al. 2018).

\section{Results}

\section{The CHUP1 mutation significantly affects maximum quantum yield of PSII under low temperature and rates of net $\mathrm{CO}_{2}$ fixation under high light}

Affected chloroplast positioning in chupl (see Supplementary Figures S1 and S2) had significant effects on photosynthesis both under ambient and low temperature. While Fv/Fm did not differ between chupl and Col-0 under control conditions, it was lower in chupl than in Col- 0 at $4{ }^{\circ} \mathrm{C}$ under both ambient and low light (Fig. 1; AL/LT: $p=0.07$; LL/LT: $p<0.05$, ANOVA). Interestingly, in both genotypes $\mathrm{Fv} / \mathrm{Fm}$ was significantly higher under LL/LT $(>0.82)$ than under control $(\sim 0.815)$ conditions ( $p<0.001$, ANOVA). In summary, maximum quantum yield of PSII did not differ between both genotypes under control conditions but was lower in chupl than in Col- 0 after 7 days at $4{ }^{\circ} \mathrm{C}$ under both low $\left(20 \mu \mathrm{mol} \mathrm{m} \mathrm{m}^{-2} \mathrm{~s}^{-1}\right)$ and ambient light $\left(100 \mu \mathrm{mol} \mathrm{m} \mathrm{m}^{-2} \mathrm{~s}^{-1}\right)$.

Rates of net $\mathrm{CO}_{2}$ assimilation were recorded together with chlorophyll fluorescence parameters within light response curves and $\mathrm{CO}_{2}$ response curves (Fig. 2, Supplementary Figures S3 and S4). Analysis of variance (ANOVA) revealed a highly significant condition effect $(p<0.001)$ on net $\mathrm{CO}_{2}$ fixation rates of both genotypes separating control vs. AL/LT and control vs. LL/LT across a wide range of PAR

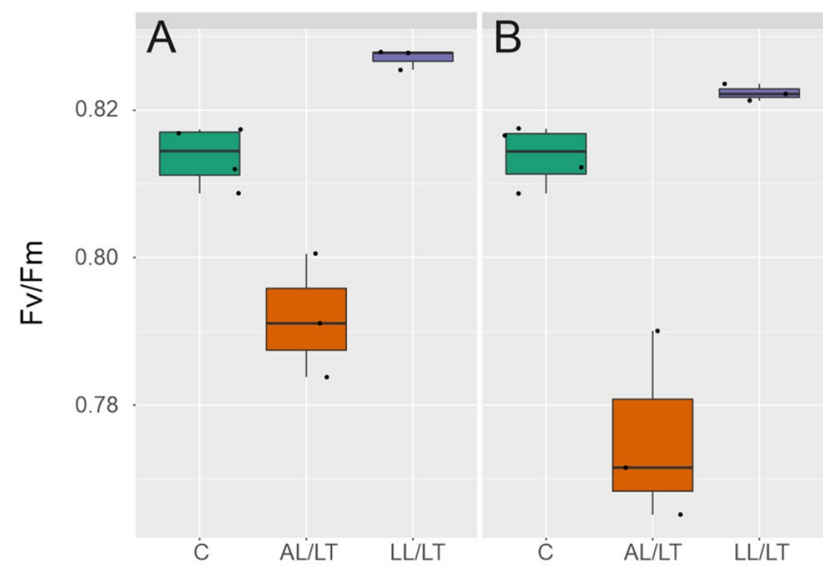

Fig. 1 Maximum quantum yield of PSII in Col-0 and chupl. a Fv/Fm in Col-0 and $\mathbf{b}$ in chupl under control (green box, c, $100 \mu \mathrm{mol} \mathrm{m} \mathrm{m}^{-2} \mathrm{~s}^{-1}, 22{ }^{\circ} \mathrm{C}$ ), ambient light/low temperature (orange box, AL/LT, $100 \mu \mathrm{mol} \mathrm{m} \mathrm{m}^{-2} \mathrm{~s}^{-1}$ ) and low light/low temperature (purple box, LL/LT, $20 \mu \mathrm{mol} \mathrm{m} \mathrm{m}^{-2} \mathrm{~s}^{-1}$ ). In addition to coloured box-andwhisker plots single measurements are indicated by black dots intensity and $\mathrm{CO}_{2}$ concentration (Fig. 2a-d). No significance was observed between AL/LT and LL/LT neither in light response curves nor in $\mathrm{CO}_{2}$ response curves. Further, under high PAR intensity, i.e. $1200 \mu \mathrm{mol} \mathrm{m} \mathrm{s}^{-2} \mathrm{~s}^{-1}$, and at ambient temperature $\left(2{ }^{\circ} \mathrm{C}\right)$ chupl had a significantly lower net $\mathrm{CO}_{2}$ fixation rate than Col-0 $(p<0.001$, ANOVA; a summary of all ANOVA results in provided in the supplements, Supplementary Tables I-III). Local polynomial regression under control conditions (green lines in Fig. 2) indicated that net $\mathrm{CO}_{2}$ assimilation rates of chupl were near to saturation at $1200 \mu \mathrm{mol} \mathrm{m} \mathrm{m}^{-2} \mathrm{~s}^{-1}$ (Fig. 2b) which was not observed for Col-0 (Fig. 2a). Under high $\mathrm{CO}_{2}$ concentration, i.e. 900 and $1200 \mathrm{ppm}$, and high irradiance $\left(1200 \mu \mathrm{mol} \mathrm{m}^{-2} \mathrm{~s}^{-1}\right)$, net $\mathrm{CO}_{2}$ assimilation rates did not significantly differ between Col-0 and chupl (Fig. 2c, d). Instead, Col-0 had significantly higher $\mathrm{CO}_{2}$ fixation rates than chupl at $200 \mathrm{ppm}$ and $400 \mathrm{ppm}$ (ANOVA, $p<0.01$ ). After $7 \mathrm{~d}$ at $4{ }^{\circ} \mathrm{C}, \mathrm{CO}_{2}$ assimilation rates were found to neither differ between genotypes nor between light conditions within a genotype, i.e. AL/LT and LL/LT.

Light and $\mathrm{CO}_{2}$ response curves of chlorophyll fluorescence parameters were recorded together with net $\mathrm{CO}_{2}$ assimilation rates (Supplementary Figures S3 and S4) revealing no significant difference between Col-0 and chup I (Supplementary Table 1). Yet, only in Col-0 qN was significantly higher under control than under low temperature at high PAR intensity, i.e. $1200 \mu \mathrm{mol} \mathrm{m}{ }^{-2} \mathrm{~s}^{-1}$ (ANOVA, $p<0.05$; Supplementary Figure 3G).

\section{Low temperature induced accumulation of hexose phosphates is significantly affected in chup 1}

Quantification of central carbohydrates revealed similar amounts of starch and sucrose in Col-0 and chupl under all tested conditions (Fig. 3). Compared to control condition, in both genotypes, starch increased significantly $\sim$ tenfold under AL/LT (ANOVA, $p<0.001$ ) and decreased significantly to a level $\sim 0.5 \mu \mathrm{mol} \mathrm{gFW}^{-1}$ under LL/LT (ANOVA, $p<0.05$; Fig. 3a, b). Similarly, sucrose increased significantly 2.5-fold under AL/LT (ANOVA, $p<0.001$ ) while no significant change was observed under LL/LT neither for Col-0 nor for chupl (Fig. 3c, d). Although it is difficult to estimate biological variance from 5 biological replicates, our data indicated a slightly higher variance of starch and sucrose amount in chupl than in Col-0, particularly under control and AL/LT. Next, we quantified dynamics of free hexoses, i.e. glucose and fructose, and of their phosphorylation products, G6P and F6P (Fig. 4). In both genotypes, glucose and fructose significantly increased under AL/LT $(p<0.001)$ and significantly dropped under LL/LT $(p<0.05)$ compared to the amount under control conditions (Fig. 4a-d). Neither amount of glucose nor fructose significantly differed between Col-0 and chupl 
Fig. 2 Rates of net $\mathrm{CO}_{2}$ assimilation in Col-0 and chupl as a function of irradiance and $\mathrm{CO}_{2}$ concentration. a Rates of net $\mathrm{CO}_{2}$ assimilation in Col-0 under control (green circles), AL/LT (orange triangles) and LL/LT (purple squares) as a function of PAR, $\mathbf{b}$ rates of net $\mathrm{CO}_{2}$ assimilation in chupl under control (green circles), AL/LT (orange triangles) and LL/LT (purple squares) as a function of PAR, $c$ rates of net $\mathrm{CO}_{2}$ assimilation in Col-0 under control (green circles), AL/LT (orange triangles) and LL/LT (purple squares) as a function of $\mathrm{CO}_{2}$ concentration, $\mathbf{d}$ rates of net $\mathrm{CO}_{2}$ assimilation in chupl under control (green circles), AL/LT (orange triangles) and LL/LT (purple squares) as a function of $\mathrm{CO}_{2}$ concentration. Symbols represent measurements of independent biological replicates $(n \geq 3)$. Lines represent a local polynomial regression. Measurements of control samples were performed at $22{ }^{\circ} \mathrm{C}$, measurements of AL/ LT and LL/LT at $4{ }^{\circ} \mathrm{C}$. An overview of significances revealed by ANOVA is provided in the supplements (Supplementary Tables I and II)
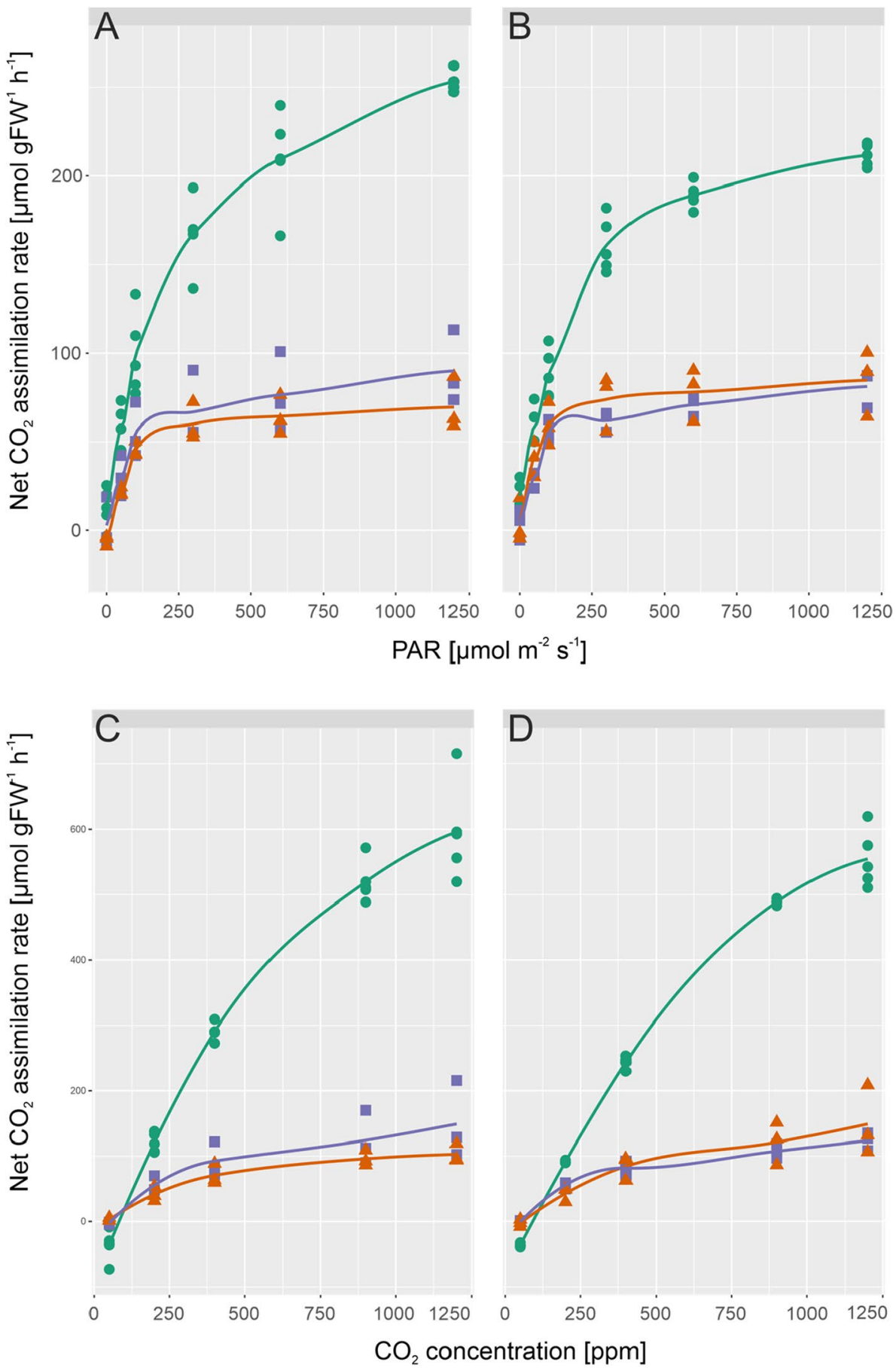

under any tested condition. In contrast, dynamics of G6P and F6P revealed significant genotype-effects under low temperature (Fig. 4e-h). While G6P amounts significantly increased $\sim$ threefold in both genotypes under AL/LT, only in Col-0 we observed a significant difference between G6P amount under AL/LT and LL/LT (Fig. 4e, f). In chupl, G6P amount was similar in LL/LT and AL/LT (Fig. 4f). Also, dynamics of F6P amount significantly differed between Col-0 and chupl where already under control conditions a reduced amount was observed in chupl ( $p=0.06$, ANOVA). Under AL/LT, amount of F6P significantly increased $\sim$ threefold in chupl while no change was observed in Col-0 (Fig. 4g, h). Only under LL/LT, F6P amount increased $\sim 1.5$-fold in Col-0 $(p<0.001)$. In summary, sucrose/starch ratios and free hexose amounts were similar in both genotypes while hexose phosphates significantly differed in their cold-induced dynamics between Col-0 and chupl. 


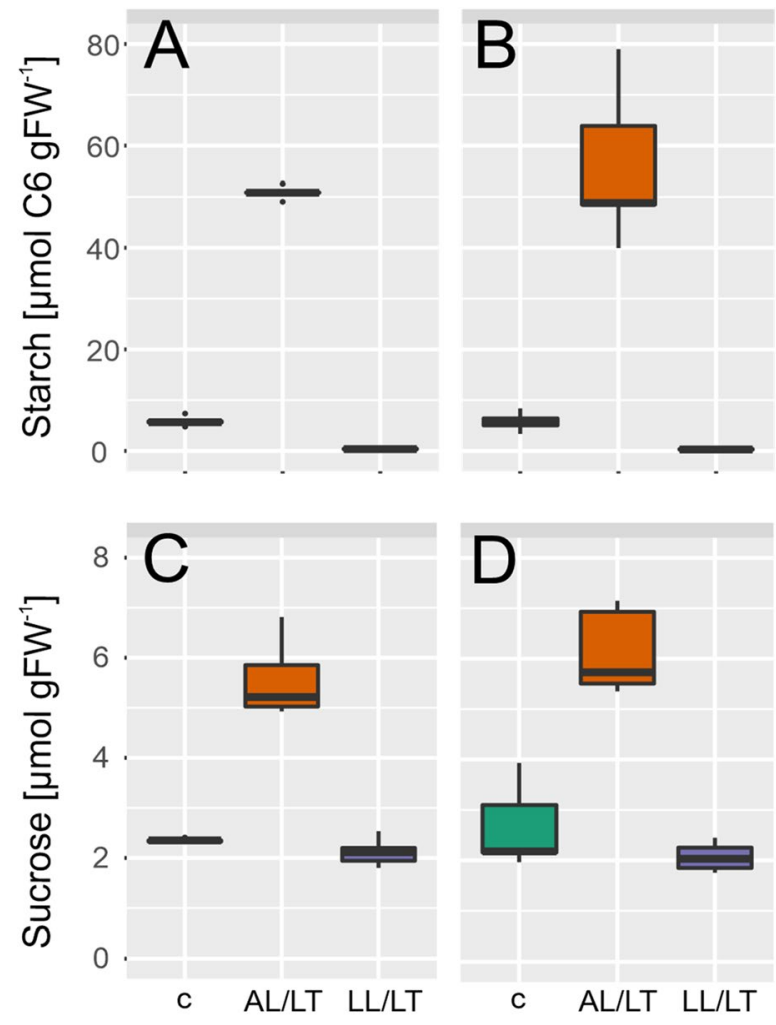

Fig. 3 Cold-induced dynamics of starch and sucrose. a Starch amount in Col-0, b Starch amount in chup1, c Sucrose amount in Col-0 and d Sucrose amount in chupl. In each panel, left/green: control; middle/ orange: AL/LT; right/purple: LL/LT. An overview of significances revealed by ANOVA is provided in the supplements (Supplementary Table III; $n=5$ )

\section{Cold-induced dynamics of gluco- and fructokinase activities are significantly reduced in chup1}

Enzyme activities of the central carbohydrate metabolism were quantified to reveal a potential effect of the CHUPI mutation on metabolic regulation (Fig. 5). Dynamics of sucrose phosphate synthase (SPS) activity under AL/LT was reduced in chupl (Fig. 5a, b). Most significant effects were observed for gluco- (GlcK) and fructokinase (FrcK) activities under low temperature (Fig. 5c-f). In Col-0, GlcK activity significantly increased $\sim$ threefold under AL/ LT compared to control conditions while no effect was observed in chupl (Fig. 5c, d). Under LL/LT, FrcK activity dropped in both genotypes but only in Col-0 this effect was significant (Fig. 5e). Activities of neutral, acidic and cell wall-associated invertases were reduced significantly under low temperature in both genotypes (see ANOVA results in Supplementary Table III) and this reduction was most pronounced under AL/LT (Fig. 5g-1). Activities of phosphoglucoisomerase (PGI) and glucose 6-phosphate dehydrogenase revealed similar cold-induced changes in both genotypes with an activity increase under AL/LT and no change or only a slight decrease under LL/LT (Supplementary Figure S5).

\section{Kinetic modelling reveals a shift of reaction rates in chup1 from hexose phosphorylation into G6P oxidation under low temperature}

Metabolite concentrations, net rates of $\mathrm{CO}_{2}$ assimilation and enzyme activities were integrated into a mathematical kinetic model. Graphical model structure and identified steady state models for Col-0 and chupl under all tested conditions are provided in the supplement (Supplemental Figure S6 and Supplemental Tables IV-VI). For kinetic simulation, a steady state was identified by parameter optimization using temperature-corrected enzyme activities and mean values of quantified metabolites. Particularly under AL/LT, simulations revealed that reaction rates of PGI and G6PDH in Col-0 decreased stronger than in chupl (Fig. 6). Vice versa, reaction rates of hexose phosphorylation ('rGLCK' and 'rFRCK') together with sucrose cleavage ('rINV') were lower in chupl than in Col-0 AL/LT. Simulated rates of sucrose biosynthesis ('rSPS') decreased to similar values in both genotypes. In summary, kinetic simulations suggest that particularly under AL/LT a higher rate of glucose oxidation, catalysed by G6PDH, in chupl leads to reduced rates of hexose phosphorylation when compared to Col-0.

\section{Discussion}

Chloroplast avoidance movement is a protective mechanism by which plants mitigate photodamage. Under high light intensities, chloroplasts typically move to the side walls of cells to minimize the area of potential light absorption. Mutants like chupl, which are defective in this avoidance movement, have been shown to be more susceptible to damage in high light than wild type plants which immediately proves the central importance of this mechanism under changing environmental conditions (Kasahara et al. 2002). However, under ambient light, i.e. $100 \mu \mathrm{mol}$ photons $\mathrm{m}^{-2} \mathrm{~s}^{-1}$, and long day growth conditions neither we (data not shown) nor others observed any effect on biomass accumulation (Kasahara et al. 2002). In contrast, for plants grown under short day conditions the accumulation response, which is also defective in chupl mutants, was shown to enhance leaf photosynthesis and plant biomass production (Gotoh et al. 2018). In line with these observations, we found similar rates of net $\mathrm{CO}_{2}$ assimilation in Col-0 and chupl at growth PAR intensity under ambient and low temperature (see Fig. 2a) while Gotoh and colleagues observed a significantly reduced rate of net $\mathrm{CO}_{2}$ assimilation in chupl grown under short day. Together with the finding of lowered net $\mathrm{CO}_{2}$ assimilation rates in chupl under high light intensity, 


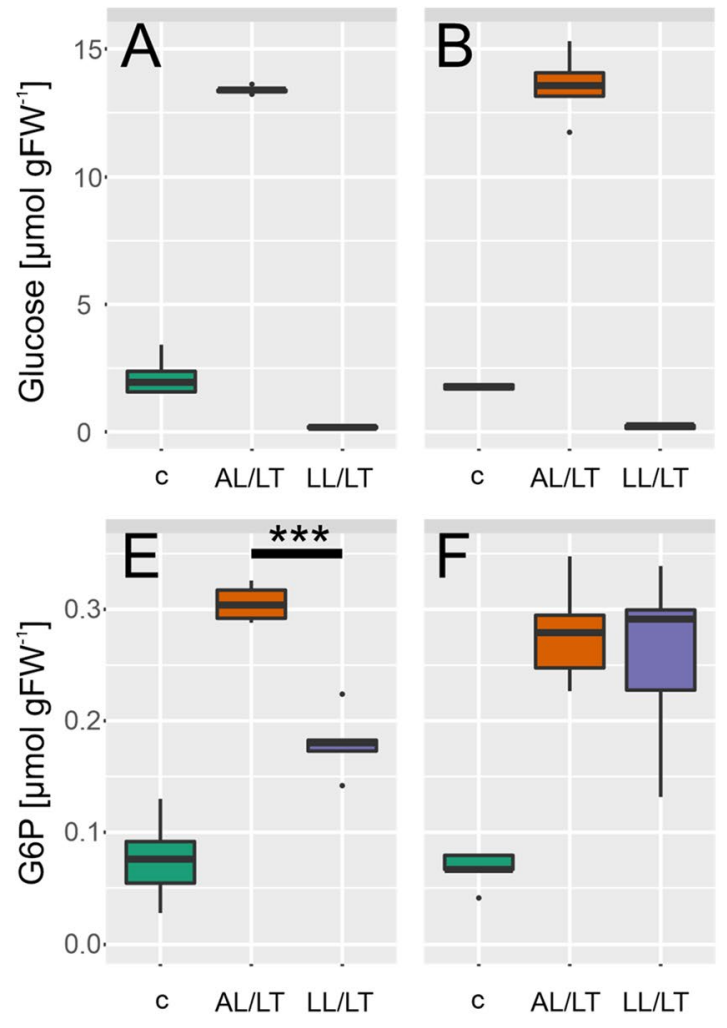

Fig. 4 Cold-induced dynamics of hexoses and hexose phosphates. a, $\mathbf{c}, \mathbf{e}, \mathbf{g}$ amount of substance in Col- $0, \mathbf{b}, \mathbf{d}, \mathbf{f}, \mathbf{h}$ amount of substance in chup1. Boxes in each panel, left/green: control; middle/orange: AL/LT; right/purple: LL/LT. Significances are indicated by asterisks
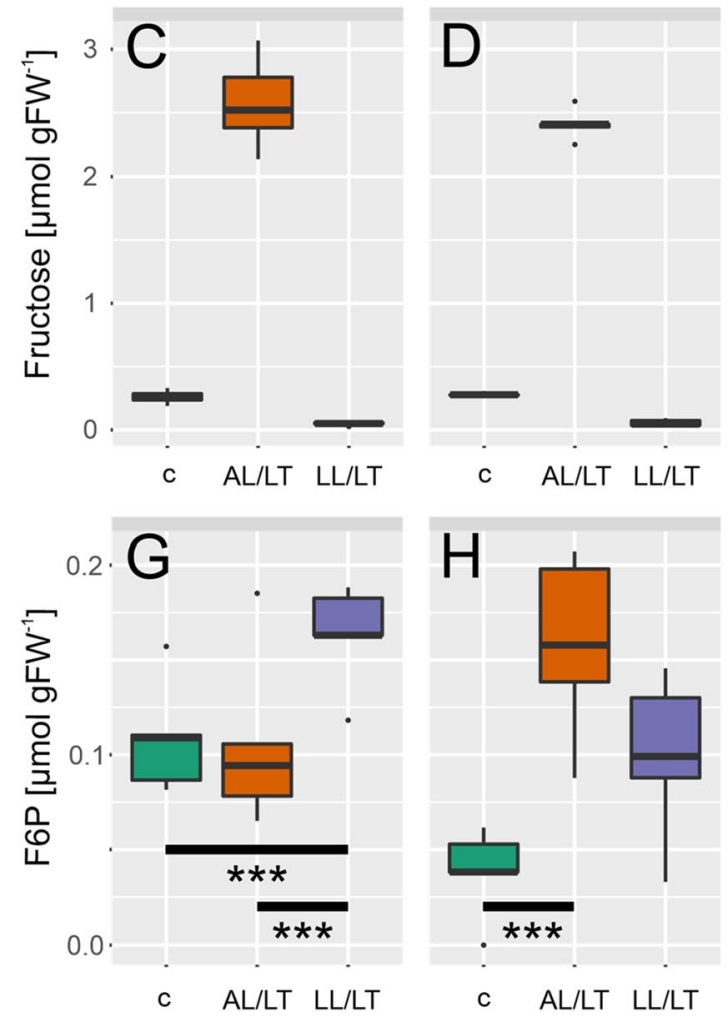

but only shown where different between Col-0 and chupl (ANOVA, $* * * p<0.001)$. A complete overview of significances is provided in the supplements (Supplementary Table III; $n=5$ )
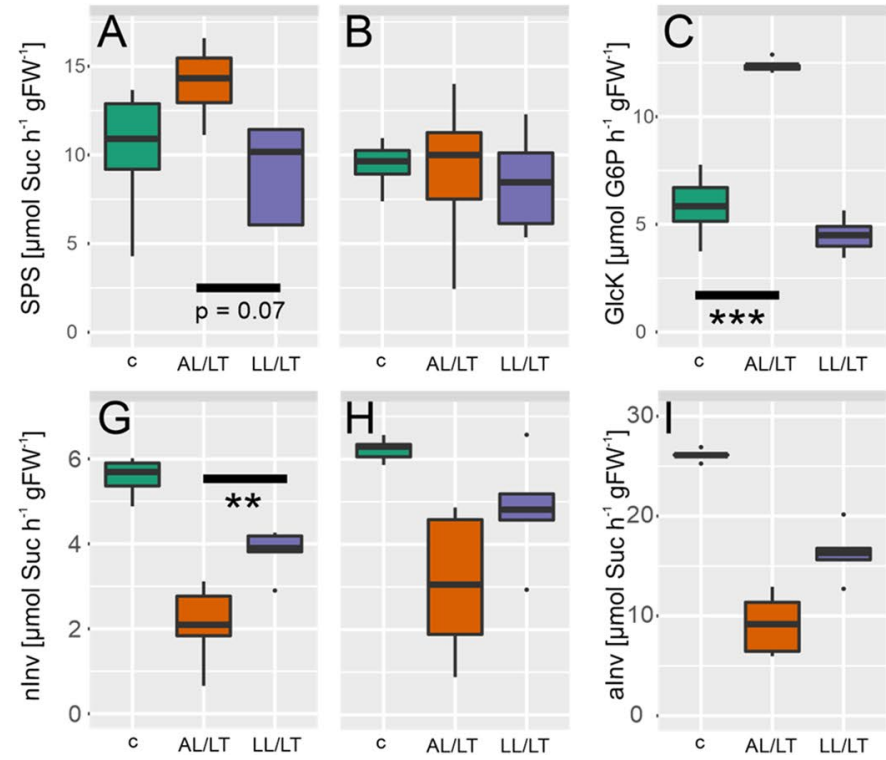

Fig. 5 Dynamics of enzyme activities of the central carbohydrate metabolism. a, c, e, g, i, $\mathbf{k}$ enzyme activities in Col-0, b, d, $\mathbf{f}, \mathbf{h}, \mathbf{j}$, $\mathbf{l}$ enzyme activities in chup1. Boxes in each panel, left/green: control; middle/orange: AL/LT; right/purple: LL/LT. SPS Sucrose phosphate synthase, GlcK glucokinase, $F r c K$ fructokinase, nInv neutral
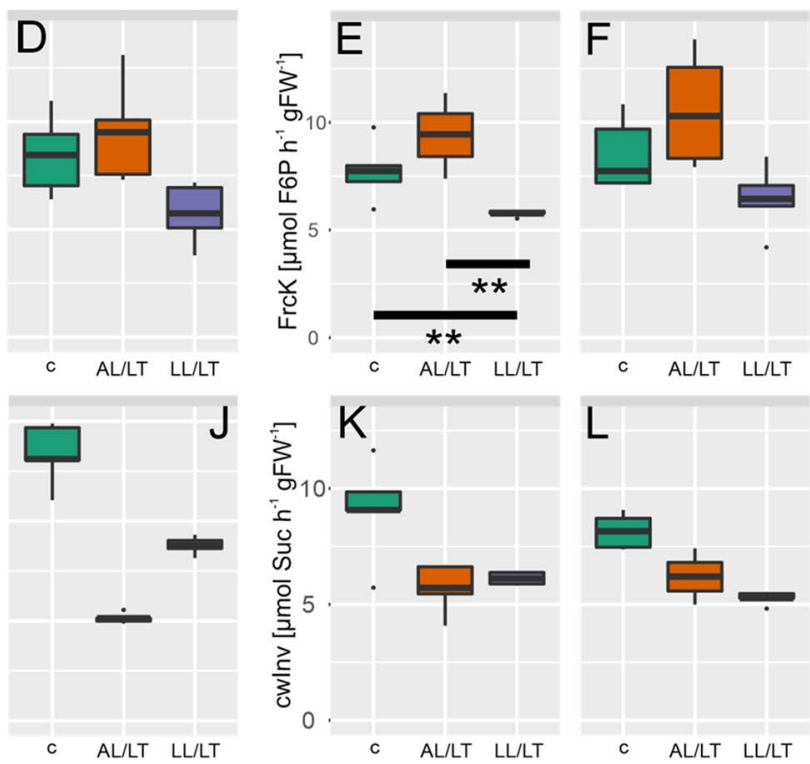

invertase, aInv acidic invertase, $c w I n v$ cell wall-associated invertase Significances are indicated by asterisks but only shown where different between Col-0 and chupl (ANOVA; ** $p<0.01$; *** $p<0.001$ ). A complete overview of significances is provided in the supplements (Supplementary Table III; $n=5$ ) 


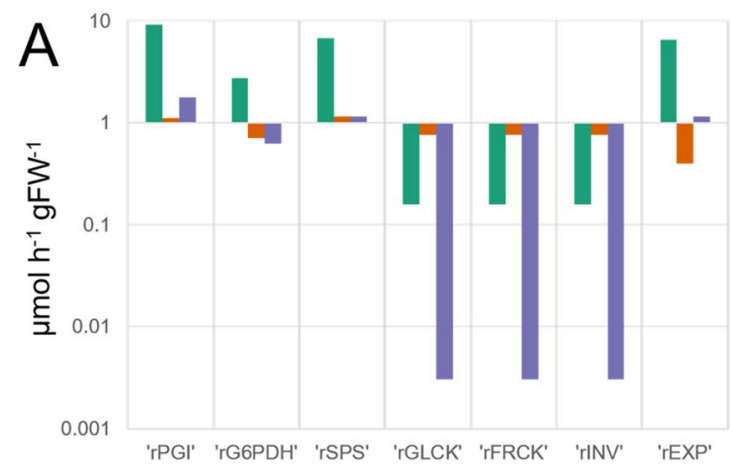

Fig. 6 Simulated reaction rates before and after cold acclimation in Col-0 and chupl. a Reaction rates of Col-0 under control, AL/LT and LL/LT. b Reaction rates of chupl under control, AL/LT and LL/LT. Green bars: control; orange bars: AL/LT; purple bars: LL/LT. Simu-

i.e. $1200 \mu \mathrm{mol}$ photons $\mathrm{m}^{-2} \mathrm{~s}^{-1}$, these results indicate that chup1 plants can compensate for effects on photosynthetic $\mathrm{CO}_{2}$ uptake rates under long photoperiods.

Under control long day growth conditions, neither of the recorded chlorophyll fluorescence parameters significantly differed between Col-0 and chupl which was also observed previously under short day growth conditions (Gotoh et al. 2018). This suggests that potential limitation of $\mathrm{CO}_{2}$ net assimilation rates in chupl rather arises from biochemistry than photochemistry. Supportively, chupl assimilated significantly less $\mathrm{CO}_{2}$ than Col-0 under ambient temperature, high light intensity and lowered $\mathrm{CO}_{2}$ concentration $(200 \mathrm{ppm}$, Fig. $2 \mathrm{~b}$ and Supplementary Table II). While under very low $\mathrm{CO}_{2}$ concentrations, i.e. $50 \mathrm{ppm}$, no significant effect was observed this might be due to the very strong overlaying effect of such a condition on all photosynthetic parameters, also in Col-0. Further evidence for a biochemical limitation of photosynthesis in chupl is provided by the finding that dynamics of enzyme activities, particularly of SPS, GlcK and Frck, were significantly lowered in chupl compared to Col-0 when exposed to $4{ }^{\circ} \mathrm{C}$ (see Fig. 5). Previous studies have shown a central role of sucrose biosynthesis for cold acclimation and stabilization of photosynthesis (Nägele et al. 2012; Strand et al. 2003). Yet, although the median SPS activity in Col-0 was $~ 1.5$-fold higher than in chupl under AL/LT, neither simulated steady state SPS flux nor Suc amount differed between both genotypes (Figs. 3 and 6). This might be explained by the significantly higher amount of F6P in chupl than in Col-0 under AL/LT (see Fig. 4). Together with UDP glucose, F6P is substrate for the SPS reaction and, thus, our data suggest that a higher amount of F6P may compensate for a reduced SPS activity to result in a similar rate of Suc biosynthesis (rSPS) in both genotypes. However, our modelling approach comprised simplifications which need to be discussed and addressed in further studies in order to reveal how far estimated reaction rates reflect

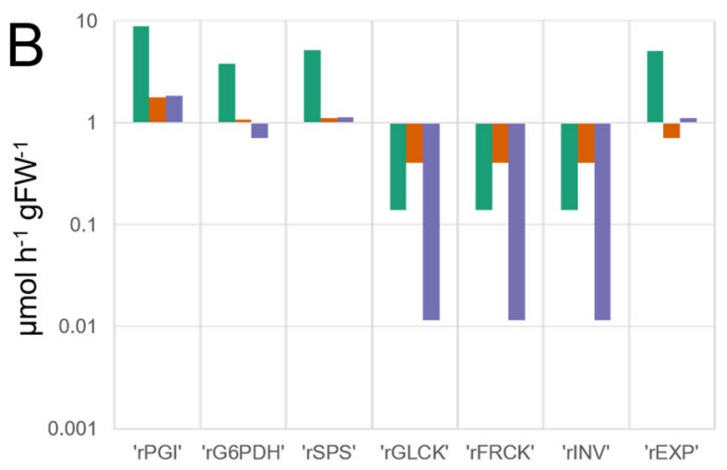

lations were derived from a kinetic model which is explained in the supplements (Supplementary Figure S6, Supplementary Table IV). Enzyme activities were temperature corrected to $4{ }^{\circ} \mathrm{C}$ for $\mathrm{AL} / \mathrm{LT}$ and LL/LT simulations

actual in vivo rates. First, in the present study we did not quantify UDP glucose but instead assumed its amount to be $20 \%$ of G6P as reported before (Szecowka et al. 2013). Further, we ignored subcellular distribution of metabolites and, thus, simulated reaction rates might overestimate actual reaction rates due to a higher substrate concentration. For example, approximately one third of F6P amount has previously been reported to be located in the chloroplast of Col-0 and two thirds are located in the cytosol (Szecowka et al. 2013). Future studies on subcellular metabolite distribution might refine the estimated reaction rates and reveal whether compartmentation differs between Col-0 and chupl.

While rSPS was similar in both genotypes under all conditions, rates of sucrose cleavage (rINV), fructose phosphorylation (rFRCK) and glucose phosphorylation (rGLCK) were lower in chupl than in Col-0 under control and AL/LT conditions (see Fig. 6). Together with rSPS, these reactions describe a cyclic reaction of Suc synthesis and degradation which has been termed as a futile cycle due to its seemingly futile consumption of ATP (Geigenberger and Stitt 1991; Nguyen-Quoc and Foyer 2001). However, it has also been discussed earlier that the "waste" of ATP might only comprise 3-5\% while, as a benefit, such futile cycles increase sensitivity of metabolic regulation (Geigenberger and Stitt 1991; Hue 1981). Also, in our own previous studies we found evidence for a central role of sucrose cycling in stabilization of photosynthesis during cold exposure (Nägele and Heyer 2013; Weiszmann et al. 2018). Interestingly, while most of the recorded photosynthetic parameters under AL/ LT were similar in both genotypes, maximum quantum yield was significantly affected, i.e. lower, in chupl and, thus, correlated with the sucrose cycling capacities determined by reaction rates rINV, rGLCK and rFRCK. Conclusively, although chupl did not suffer from severe photodamage under AL/LT, our findings provide evidence for an important role of sucrose cycling in precision tuning of photosynthesis 
and metabolism under low temperature. This observation emphasizes not only the suitability but also the necessity of mathematical modelling to reveal effects of mutations and/ or environmental fluctuation on metabolism. Particularly for the study of metabolic cycles mathematical modelling is the method of choice due to underlying complexity, nested uncertainties and non-intuitive modes of regulation (Henkel et al. 2011; Reznik and Segrè 2010; Schaber et al. 2009).

In addition to activity of hexokinase, oxidation of G6P, catalysed by G6PDH, represents a further important mechanism involved in dynamics of hexose phosphate concentrations under changing environmental conditions. Cold acclimation under AL/LT resulted in a threefold increase of the G6P/F6P ratio in Col-0 and only $\sim 1.5$-fold in chupl. Vice versa, under LL/LT this ratio increased $\sim$ threefold in chup 1 compared to control while in Col-0 concentrations of G6P and F6P increased similarly. A significant increase of the G6P/F6P ratio due to cold acclimation has been reported earlier (Savitch et al. 2001). As suggested by Savitch and colleagues, induction of the ascorbate-glutathione cycle as a ROS defence system might result in an increased supply of $\mathrm{NADPH}+\mathrm{H}^{+}$by an increased activity of the oxidative pentose phosphate pathway, oxPPP (May et al. 1998; Savitch et al. 2001). In chupl, both G6P and F6P amount significantly increased under AL/LT which together with the wild type-like activities of G6PDH and no obvious photodamage might indicate that ROS defence via oxPPP is still functional. Yet, overaccumulation of G6P under LL/ LT and higher rG6PDH rates indicate a less tightly regulated balance between photosynthetic light reactions and carbohydrate metabolism in chupl which, under high light intensities, would result in lowered $\mathrm{CO}_{2}$ assimilation rates and photodamage.

\section{Conclusion}

Although cold acclimation of chupl did not result in a lowered photosynthetic $\mathrm{CO}_{2}$ assimilation rate compared to Col-0, the presented findings suggest a central role of CHUP1-mediated chloroplast positioning for tight regulation of photosynthesis and carbohydrate metabolism under low temperature. Future studies might address metabolic effects on the subcellular level, including further mutants affected in chloroplast positioning, e.g. phototropin mutants. While the combination of high light intensities and low temperature leads to photoinhibition which prevents the study of metabolic regulation under such conditions, further insights into the effects of chloroplast positioning on metabolic regulation might be gained using other abiotic factors, e.g. heat, high light and a combination of both.
Acknowledgements We thank the groups of Plant Evolutionary Cell Biology and Plant Development at LMU München for many fruitful discussions and support.

Author contributions AK performed experiments, KS performed microscopy, LF, LS, TS, SF and TN supported experimental analysis. AK and TN analysed data and wrote the manuscript. All authors approved the manuscript.

Funding Open Access funding enabled and organized by Projekt DEAL. This work was supported by the LMUexcellent Junior Researcher Fund and by the TRR175, funded by Deutsche Forschungsgemeinschaft (DFG).

\section{Compliance with ethical standards}

Conflict of interest The authors declare that they have no conflict of interest.

Open Access This article is licensed under a Creative Commons Attribution 4.0 International License, which permits use, sharing, adaptation, distribution and reproduction in any medium or format, as long as you give appropriate credit to the original author(s) and the source, provide a link to the Creative Commons licence, and indicate if changes were made. The images or other third party material in this article are included in the article's Creative Commons licence, unless indicated otherwise in a credit line to the material. If material is not included in the article's Creative Commons licence and your intended use is not permitted by statutory regulation or exceeds the permitted use, you will need to obtain permission directly from the copyright holder. To view a copy of this licence, visit http://creativecommons.org/licenses/by/4.0/.

\section{References}

Aro E-M, Hundal T, Carlberg I, Andersson B (1990) In vitro studies on light-induced inhibition of Photosystem II and D1-protein degradation at low temperatures. BBA Bioenergetics 1019:269-275. https://doi.org/10.1016/0005-2728(90)90204-H

Bahrani H et al (2019) Preferential accumulation of glycosylated cyanidins in winter-hardy rye (Secale cereale L.) genotypes during cold acclimation. Environ Exp Bot 164:203-212. https://doi. org/10.1016/j.envexpbot.2019.05.006

Dreyer A, Dietz K (2018) Reactive oxygen species and the redox-regulatory network in cold stress acclimation. Antioxidants 7:169. https://doi.org/10.3390/antiox7110169

Fahrendorf T, Ni B, Shorrosh BS, Dixon RA (1995) Stress responses in alfalfa (Medicago sativa L.) XIX. Transcriptional activation of oxidative pentose phosphate pathway genes at the onset of the isoflavonoid phytoalexin response. Plant Mol Biol 28(5):885-900

Fürtauer L, Nägele T (2016) Approximating the stabilization of cellular metabolism by compartmentalization. Theory Biosci 135:73-87. https://doi.org/10.1007/s12064-016-0225-y

Fürtauer L, Weiszmann J, Weckwerth W, Nägele T (2019) Dynamics of plant metabolism during cold acclimation. Int J Mol Sci 20:5411

Geigenberger P, Stitt M (1991) A futile cycle of sucrose synthesis and degradation is involved regulating partitioning between sucrose starch and respiration in cotyledons of germinating ricinus-communis 1 . Seedlings when phloem transport is inhibited. Planta 185:81-90

Gibon Y, Vigeolas H, Tiessen A, Geigenberger P, Stitt M (2002) Sensitive and high throughput metabolite assays for inorganic pyrophosphate, ADPGlc, nucleotide phosphates, and glycolytic 
intermediates based on a novel enzymic cycling system. Plant $\mathrm{J}$ 30:221-235

Gotoh E et al (2018) Chloroplast accumulation response enhances leaf photosynthesis and plant biomass production. Plant Physiol 178:1358-1369. https://doi.org/10.1104/pp.18.00484

Gururani Mayank A, Venkatesh J, Tran LSP (2015) Regulation of photosynthesis during abiotic stress-induced photoinhibition. Mol Plant 8:1304-1320. https://doi.org/10.1016/j.molp.2015.05.005

Henkel S, Nägele T, Hörmiller I, Sauter T, Sawodny O, Ederer M, Heyer AG (2011) A systems biology approach to analyse leaf carbohydrate metabolism in Arabidopsis thaliana. EURASIP J Bioinform Syst Biol 2011:2. https://doi.org/10.1186/1687-4153-2011-2

Herrmann HA, Schwartz JM, Johnson GN (2019) Metabolic acclimation-a key to enhancing photosynthesis in changing environments? J Exp Bot 70:3043-3056. https://doi.org/10.1093/jxb/erz157

Hoffmann MH (2002) Biogeography of Arabidopsis thaliana (L.) Heynh. (Brassicaceae). J Biogeogr 29:125-134. https://doi.org/ 10.1046/j.1365-2699.2002.00647.x

Hue L (1981) The role of futile cycles in the regulation of carbohydrate metabolism in the liver. In: (Ed.). AM (ed) Advances in Enzymology and Related Areas of Molecular Biology. pp 247-331. https:// doi.org/https://doi.org/10.1002/9780470122976.ch4

Johnson HS (1972) Dithiothreitol: An inhibitor of glucose-6-phosphate-dehydrogenase activity in leaf extracts and isolated chloroplasts. Planta 106:273-277. https://doi.org/10.1007/BF00388105

Kasahara M, Kagawa T, Oikawa K, Suetsugu N, Miyao M, Wada M (2002) Chloroplast avoidance movement reduces photodamage in plants. Nature 420:829-832. https://doi.org/10.1038/nature01213

Khanal N, Bray GE, Grisnich A, Moffatt BA, Gray GR (2017) Differential mechanisms of photosynthetic acclimation to light and low temperature in Arabidopsis and the extremophile Eutrema salsugineum. Plants 6:32. https://doi.org/10.3390/plants6030032

Kunz H-H, Zamani-Nour S, Hausler RE, Ludewig K, Schroeder JI, Malinova I, Fettke J, Flugge U-I, Gierth M (2014) Loss of cytosolic phosphoglucose isomerase affects carbohydrate metabolism in leaves and is essential for fertility of Arabidopsis. Plant Physiol 166(2):753-765

Levitt J (1980) Responses of plants to environmental stresses, 2nd edn. Academic Press, New York

Liu Y, Dang P, Liu L, He C (2019) Cold acclimation by the CBF-COR pathway in a changing climate: lessons from Arabidopsis thaliana. Plant Cell Rep 38:511-519. https://doi.org/10.1007/s0029 9-019-02376-3

May MJ, Vernoux T, Leaver C, Montagu MV, Inze D (1998) Glutathione homeostasis in plants: implications for environmental sensing and plant development. J Exp Bot 49:649-667

Nägele T, Henkel S, Hörmiller I, Sauter T, Sawodny O, Ederer M, Heyer AG (2010) Mathematical modeling of the central carbohydrate metabolism in Arabidopsis reveals a substantial regulatory influence of vacuolar invertase on whole plant carbon metabolism. Plant Physiol 153:260-272. https://doi.org/10.1104/pp.110.15444 3

Nägele T, Heyer AG (2013) Approximating subcellular organisation of carbohydrate metabolism during cold acclimation in different natural accessions of Arabidopsis thaliana. New Phytol 198:777787. https://doi.org/10.1111/nph.12201

Nägele T, Stutz S, Hörmiller II, Heyer AG (2012) Identification of a metabolic bottleneck for cold acclimation in Arabidopsis thaliana. Plant J 72:102-114. https://doi.org/10.1111/j.1365313X.2012.05064.X

Nguyen-Quoc B, Foyer CH (2001) A role for "futile cycles" involving invertase and sucrose synthase in sucrose metabolism of tomato fruit. J Exp Bot 52:881-889

Oikawa K et al (2003) Chloroplast unusual positioning1 is essential for proper chloroplast positioning. Plant Cell 15:2805-2815. https:// doi.org/10.1105/tpc.016428
Oikawa K et al (2008) Chloroplast outer envelope protein CHUP1 is essential for chloroplast anchorage to the plasma membrane and chloroplast movement. Plant Physiol 148:829-842. https://doi. org/10.1104/pp.108.123075

Patzke K et al (2019) The plastidic sugar transporter pSuT influences flowering and affects cold responses. Plant Physiol 179:569-587. https://doi.org/10.1104/pp.18.01036

Pons TL (2012) Interaction of temperature and irradiance effects on photosynthetic acclimation in two accessions of Arabidopsis thaliana. Photosynth Res 113(1-3):207-219

R Core Team (2019) R: a language and environment for statistical computing. R Foundation for Statistical Computing, Vienna, Austria

Reyes BA, Pendergast JS, Yamazaki S (2008) Mammalian peripheral circadian oscillators are temperature compensated. J Biol Rhythms 23:95-98. https://doi.org/10.1177/0748730407311855

Reznik E, Segrè D (2010) On the stability of metabolic cycles. J Theor Biol 266:536-549. https://doi.org/10.1016/j.jtbi.2010.07.023

RStudio Team (2019) RStudio: integrated Development for R. RStudio Inc, Boston, MA

Ruan YL (2014) Sucrose metabolism: gateway to diverse carbon use and sugar signaling. Annu Rev Plant Biol 65:33-67. https://doi. org/10.1146/annurev-arplant-050213-040251

Savitch LV, Barker-Astrom J, Ivanov AG, Hurry V, Oquist G, Huner NP, Gardeström P (2001) Cold acclimation of Arabidopsis thaliana results in incomplete recovery of photosynthetic capacity, associated with an increased reduction of the chloroplast stroma. Planta 214:295-303

Schaber J, Liebermeister W, Klipp E (2009) Nested uncertainties in biochemical models. IET Syst Biol 3:1-9. https://doi.org/10.1049/ iet-syb:20070042

Scharte J, Schön H, Tjaden Z, Weis E, von Schaewen A (2009) Isoenzyme replacement of glucose-6-phosphate dehydrogenase in the cytosol improves stress tolerance in plants. Proc Natl Acad Sci U S A 106:8061-8066. https://doi.org/10.1073/pnas.0812902106

Schmidt von Braun S, Schleiff E (2008) The chloroplast outer membrane protein CHUP1 interacts with actin and profilin. Planta 227:1151-1159. https://doi.org/10.1007/s00425-007-0688-7

Schnarrenberger C, Oeser A (1974) Two isoenzymes of glucosephosphate isomerase from spinach leaves and their intracellular compartmentation. Eur J Biochem 45:77-82. https://doi. org/10.1111/j.1432-1033.1974.tb03531.x

Stitt M, Lunn J, Usadel B (2010) Arabidopsis and primary photosynthetic metabolism-more than the icing on the cake. Plant J 61:1067-1091. https://doi.org/10.1111/j.1365-313X.2010.04142 .X

Strand A, Foyer CH, Gustafsson P, Gardeström P, Hurry V (2003) Altering flux through the sucrose biosynthesis pathway in transgenic Arabidopsis thaliana modifies photosynthetic acclimation at low temperatures and the development of freezing tolerance. Plant Cell Environ 26:523-535

Strand A, Zrenner R, Trevanion S, Stitt M, Gustafsson P, Gardestrom $P(2000)$ Decreased expression of two key enzymes in the sucrose biosynthesis pathway, cytosolic fructose-1,6-bisphosphatase and sucrose phosphate synthase, has remarkably different consequences for photosynthetic carbon metabolism in transgenic Arabidopsis thaliana. Plant J 23:759-770. https://doi.org/10.1046/ j.1365-313x.2000.00847.x

Szecowka M et al (2013) Metabolic fluxes in an illuminated Arabidopsis rosette. Plant Cell 25:694-714. https://doi.org/10.1105/ tpc.112.106989

Wada M (2013) Chloroplast movement. Plant Sci 210:177-182. https ://doi.org/10.1016/j.plantsci.2013.05.016

Wang L et al (2017) The inhibition of protein translation mediated by AtGCN1 is essential for cold tolerance in Arabidopsis thaliana. Plant Cell Environ 40:56-68. https://doi.org/10.1111/pce.12826 
Wanner LA, Junttila O (1999) Cold-induced freezing tolerance in Arabidopsis. Plant Physiol 120:391-400

Weiszmann J, Fürtauer L, Weckwerth W, Nägele T (2018) Vacuolar sucrose cleavage prevents limitation of cytosolic carbohydrate metabolism and stabilizes photosynthesis under abiotic stress. FEBS J 285:4082-4098. https://doi.org/10.1111/febs.14656

Wiese A et al (1999) Spinach hexokinase I is located in the outer envelope membrane of plastids. FEBS Lett 461:13-18
Xin Z, Browse J (2000) Cold comfort farm: the acclimation of plants to freezing temperatures. Plant Cell Environ 23:893-902

Publisher's Note Springer Nature remains neutral with regard to jurisdictional claims in published maps and institutional affiliations. 